\title{
Monotonicity and Continuity
}

\author{
Morisuke Hasumi* and Kôzô YaButa*
}

1. It is an elementary but useful fact that a real monotone function $f(x)$ on an interval admits one-sided limits $f(x \pm 0)$ at every point $x$ in the interval and has an at most countable number of discontinuities. The same property is therefore shared with every function of bounded variation. The purpose of this rather expository note is to point out that the property is possessed by a still wider class of functions. Our observation rests on the following simple

Proposition 1. Let $h$ be a positive real function on $(0,+\infty)$ such that $h(t) \rightarrow 0$ as $t \rightarrow 0$. Suppose that $f$ is a real function defined on an interval $(a, b)$, $a<b$, and satisfies the condition

$$
f\left(x^{\prime \prime}\right)-f\left(x^{\prime}\right) \leqq h\left(x^{\prime \prime}-x^{\prime}\right)
$$

for any $x^{\prime}, x^{\prime \prime}$ with $a<x^{\prime}<x^{\prime \prime}<a^{\prime}<b$. Then $f(a+0)$ exists, belongs to $(-\infty$, $+\infty]$ and in fact is equal to

$$
\lim \inf \{f(x): x>a, x \rightarrow a\} .
$$

Proor. We see that $h$ is bounded in a right neighborhood of 0 . The relation (1) thus implies that $f$ is bounded from below in a right neighborhood of $a$. It follows that

$$
A=\lim \inf \{f(x): x>a, x \rightarrow a\}
$$

is either finite or $+\infty$. So there exists a sequence $\left\{x_{n}\right\}$ in $(a, b)$ tending to $a$ such that $f\left(x_{n}\right) \rightarrow A$. Suppose that $A$ is finite. Then, for any $\varepsilon>0$, there exists a $\delta$ with $0<\delta<a^{\prime}-a$ such that $h(t)<\varepsilon$ for $0<t<\delta$ and $f(x)>A-\varepsilon$ for any $x \in(a, a+\delta)$. For each $x \in(a, a+\delta)$ we can choose $x_{n}$ with $a<x_{n}<x$, so that $A-\varepsilon<f(x) \leqq$ $f\left(x_{n}\right)+h\left(x-x_{n}\right) \leqq f\left(x_{n}\right)+\varepsilon$. Letting $n \rightarrow+\infty$, we get $A-\varepsilon<f(x) \leqq A+\varepsilon$ for $x \in$ $(a, a+\delta)$. As $\varepsilon$ is arbitrary, we have $f(x) \rightarrow A$ as $x \rightarrow a+0$.

If $A=+\infty$, then we trivially have $f(x) \rightarrow+\infty$ as $x \rightarrow a+0$.

Q.E.D.

A similar argument will show the following

Proposition 1'. Let $h$ be as above. If $f$ is defined on $(c, a), c<a$, and satisfies the relation (1) for any $x^{\prime}, x^{\prime \prime}$ with $c<a^{\prime \prime}<x^{\prime}<x^{\prime \prime}<a$, then $f(a-0)$ exists

Received February 26, 1980.

* Department of Mathematics, Faculty of Science, Ibaraki University, Mito, Ibaraki 310, Japan. 
in the set $[-\infty,+\infty)$ and is equal to $\lim \sup \{f(x): x<a, x \rightarrow a\}$.

COROLLARY 1. If $f$ is defined on $(c, b)$ with $c<a<b$ and satisfies the relation (1) for any $x^{\prime}, x^{\prime \prime}$ with $x^{\prime}<x^{\prime \prime}$ belonging to a neighborhood of $a$, then the onesided limits $f(a \pm 0)$ exist and are finite. Moreover, we have $f(a+0) \leqq f(a) \leqq$ $f(a-0)$.

Corollary 2. If $f$ is defined on an open interval I and satisfies (1) in some neighborhood of each point of $I$, then $f$ is continuous in I except at a countable number of points and all the discontinuities of $f$ are of the first kind.

2. If the function $h$ is identically zero, then the functions $f$ satisfying (1) are nothing but monotone (decreasing) functions. On the other hand, by choosing $h$ in a suitable way, one can deal with functions neither monotone nor of bounded variation. Such a case appears in the theory of premeasures initiated in a paper [1] of Korenblum.

Let $\Re$ be the set of all open, closed and half-closed arcs of the unit circle $T$, including all the single points, $T$ itself and $\phi$. For every $I \in \mathfrak{R}$ we set $\kappa(I)=$ $\frac{|I|}{2 \pi}\left(\log \frac{2 \pi}{|I|}+1\right)$, where $|I|$ denotes the angular length of $I$. When $|I|=0$, we set $\kappa(I)=0$. A function $\mu: \Omega \rightarrow R$ is called a premeasure if

(i) $\mu\left(I_{1} \cup I_{2}\right)=\mu\left(I_{1}\right)+\mu\left(I_{2}\right)$ if $I_{1}, I_{2} \in \Re, I_{1} \cap I_{2}=\phi$ and $I_{1} \cup I_{2} \in \Re$;

(ii) $\mu(\boldsymbol{T})=0$;

(iii) $\lim _{n \rightarrow \infty} \mu\left(I_{n}\right)=0$ for every sequence $\left\{I_{n}\right\} \subset \Re$ such that $I_{1} \supset I_{2} \supset \cdots, \cap I_{n}$ $=\phi$.

With every premeasure $\mu$ we associate a function $\hat{\mu}$ defined by $\hat{\mu}(\theta)=\mu\left(I_{\theta}\right), 0<\theta$ $\leqq 2 \pi$, where $I_{\theta}=\{\zeta \in T: 0 \leqq \arg \zeta<\theta\}$. Since $\mu(T)=0$, we have $\mu(2 \pi)=0$ and $\beta$ can be extended to $\boldsymbol{R}$ as a function of period $2 \pi$, which we denote by the same symbol. A premeasure $\mu$ is said to be $\kappa$-bounded from above if there is a constant $C>0$ such that $\mu(I) \leqq C \kappa(I)$ for $I \in \mathfrak{R}$. We set $\kappa(t)=\frac{t}{2 \pi}\left(\log \frac{2 \pi}{t}+1\right)$ for $0<t \leqq 2 \pi$. If we wish, we extend $\kappa(t)$ to $[0,+\infty)$ by setting, for instance, $\kappa(0)=0$ and $\kappa(t)=$ $t / 2 \pi$ for $t \geqq 2 \pi$. Then the condition $\mu(I) \leqq C \kappa(I)$ is equivalent to saying that $\hat{\mu}\left(x^{\prime \prime}\right)$ $-\hat{\mu}\left(x^{\prime}\right) \leqq C \kappa\left(x^{\prime \prime}-x^{\prime}\right)$ for $x^{\prime}<x^{\prime \prime} \leqq x^{\prime}+2 \pi$. This is exactly in the class of inequalities we have considered above.

We look at the following special case of the Helly-type selection theorem due to Korenblum $[1 ; \S 4$, Theorem 1$]$.

Proposition 2. Let $\left\{\mu_{n}\right\}$ be a sequence of premeasures such that $\mu_{n}(I) \leqq$ $C \kappa(I)$ for any $I \in \mathcal{R}$ and any $n$. Then there exist a subsequence $\left\{\mu_{n(j)}\right\}$ and a premeasure $\mu$ such that $\beta_{n(j)}(\theta) \rightarrow \mu(\theta)$ at every point $\theta$ of continuity of $A$.

This may be compared with the well known classical result asserting that any uniformly bounded sequence of monotone functions has a convergent subsequence 
(cf. Widder [22 Theorem 16.2]). In fact, we can prove Proposition 2 just as in the case of the classical theorem (e.g., [2; Theorem 16.2]) by making use of our Corollary 1. Once we prove Proposition 2, this can be used to show the decomposition theorem of a premeasure of $\kappa$-bounded variation into the difference of two premeasures $\kappa$-bounded from above $([1 ; \S 4$, Theorem 5]). And a repeated use of Proposition 2 finally proves the Helly-type selection theorem of premeasures of $\kappa$-bounded variation.

\section{References}

[1] B. Korenblum, An extension of the Nevanlinna theory, Acta Math. 135 (1975), 187-219.

[2] D. V. Widder, The Laplace Transform, Plinceton Univ. Press, Princeton, 1941. 